\title{
A New Megastable Chaotic Oscillator with Blinking Oscillation terms
}

\author{
Dhinakaran Veeman, ${ }^{1}$ Hayder Natiq, ${ }^{2}$ Nadia M. G. Al-Saidi, ${ }^{3}$ Karthikeyan Rajagopal $1{ }^{4}$ \\ Sajad Jafari, ${ }^{5,6}$ and Iqtadar Hussain ${ }^{7}$ \\ ${ }^{1}$ Centre for Applied Research, Chennai Institute of Technology, Chennai, India \\ ${ }^{2}$ Information Technology Collage, Imam Ja'afar Al-Sadiq University, Baghdad 10001, Iraq \\ ${ }^{3}$ Department of Applied Sciences, University of Technology, Baghdad, Iraq \\ ${ }^{4}$ Centre for Nonlinear Systems, Chennai Institute of Technology, Chennai, India \\ ${ }^{5}$ Health Technology Research Institute, Amirkabir University of Technology, 424 Hafez Ave., Tehran 15875-4413, Iran \\ ${ }^{6}$ Department of Biomedical Engineering, Amirkabir University of Technology, 424 Hafez Ave., Tehran 15875-4413, Iran \\ ${ }^{7}$ Department of Mathematics, Statistics and Physics, Qatar University, Doha 2713, Qatar
}

Correspondence should be addressed to Karthikeyan Rajagopal; rkarthiekeyan@gmail.com

Received 25 February 2021; Revised 1 April 2021; Accepted 22 April 2021; Published 30 April 2021

Academic Editor: Jorge-Antonio Lopez-Renteria

Copyright (c) 2021 Dhinakaran Veeman et al. This is an open access article distributed under the Creative Commons Attribution License, which permits unrestricted use, distribution, and reproduction in any medium, provided the original work is properly cited.

\begin{abstract}
Recently, megastable systems have grabbed many researchers' interests in the area of nonlinear dynamics and chaotic systems. In this paper, the oscillatory terms' coefficients of the simplest megastable oscillator are forced to blink in time. The forced system can generate an infinitive number of hidden attractors without changing parameters. The behavior of these hidden attractors can be chaotic, tori, and limit cycle. The attractors' topology of the system seems unique and looks like picture frames. Besides, the existence of different coexisting attractors with different kinds of behaviors reflects the system's high sensitivity. Using the sample entropy algorithm, the system's complexity for different initial values is assessed. In addition, the circuit of the introduced forced system is designed, and the possibility of implicating the system with analog elements is investigated.
\end{abstract}

\section{Introduction}

Simple and elegant oscillators are interesting for researchers in the fields of nonlinear dynamics [1]. Faghani et al. have introduced many of these simple oscillators, generating chaotic time series [2]. An equilibrium point in the basin of attraction is considered an important feature for chaotic attractors [3]. Therefore, attractors can be classified into two main groups based on this feature: self-excited attractors and hidden attractors [4]. For self-excited attractors, at least one fixed point can be found in their basin of attraction [5]. On the other hand, no-equilibrium exists in the basin of attraction of a hidden attractor [6]. Different kinds of oscillators with hidden attractors have been introduced yet. Instances for oscillators with hidden attractors can be chaotic dynamics which have one stable equilibrium [7], a line of equilibriums [8], or a surface of equilibriums [9] in their basin. Hidden attractors' existence in real-world systems also has been demonstrated [10]. Besides the classification among attractors based on their equilibrium(s), an important category of chaotic systems is the forced dynamics (time-variant systems) [11]. As one of the oldest examples, Van der Pol forced oscillator can be mentioned [12]. Forcing nonlinear systems is a method to generate strange attractors when the original versions of nonlinear systems are unable to generate chaotic behaviors [11].

Besides properties such as the existence and topology of fixed points and dynamics' time variability, some other features have grabbed researchers' attention. Oscillators with time delays in their equations [13], fractional equations [14], fuzzy differential equations [15], those with hyperchaos [16], and others with synchronization among a group of them [17] 
can be examples of chaotic systems with specific features. Multistability is another of these features [18]. A system can be named multistable when it has more than one attractor without any change in its parameters [19]. In these systems, initial conditions determine trajectories which finally are attracted to which one of the attractors [20]. Multistability is sometimes considered a nonproper phenomenon that may make unexpected situations when it can also be used as a control strategy to switch among different attractors [21]. Multistable systems with uncountable infinitive attractors are called extreme multistable [22] when the ones with countable infinitive attractors are referred megastable [23]. The attractors of multistable systems can have chaotic or hyperchaotic behaviors [24], as well as some other features such as having a number of scrolls [25]. Multistable systems can have some applications such as secure communication [26]. Multistability is also investigated in natural phenomena such as the brain [27].

Besides the mentioned features, topology and the shape of strange attractors are considered as other important features that an oscillator may have [28]. Some chaotic dynamics with different types of symmetries have been introduced [29]. Among features related to the topology of attractors, ones which have multiscrolls are interesting for researchers [30]. Assessing the stability of multiscrolls attractors [31] and finding methods to preserve multiscrolls [32] are topics that grab much attention. Besides, some methods have been introduced to use multiscroll attractors such as switches in systems [33]. In addition, chaotic systems with some modifications sometimes have been used to generate Brownian motions [34]. These Brownian motions are also generated with fractional systems [35]. Besides all of these features, if an oscillator has simple algebraic equations, it can be its advantage [36].

Complexity is another feature that has been investigated among chaotic systems [37]. Richman et al. [38] have derived sample entropy (SamEn) from approximate entropy that is used for assessing the complexity of chaotic systems [39]. With this method, the complexity of the time series generated by the introduced system is investigated in this work.

The feasibility of chaotic dynamics has been a matter of interest since Lorenz discovered the first chaotic system [40]. To assess chaotic oscillators' feasibility, they have been simulated (with software such as Pspice [41]) and implemented (with analog circuits [42]). For instance, some fractional chaotic systems have been implicated with analog circuits [43] and/or digital circuits (such as field-programmable gate array (FPGA) [44]), and their feasibility has been shown [45]. The possibility of the implication of a chaotic system that has a multiscroll attractor has been demonstrated [46]. Besides, multistable systems are also implicated with both digital and analog circuits. For instance, the FPGA realization of a jerk multistable system has been investigated [47]. In another work, multistable systems with circles [48] or other strange curves of equilibrium points [49] have been realized using FPGA. Assessing the realization of the synchronization among chaotic systems is another matter of interest for researchers [50]. Such circuits can have different applications. Predicting the time series of chaotic systems [51], image encryption [52], secure communication [53], and random number generation [54] is an example of their application. In this paper, the introduced forced system's analog circuit is designed and simulated with Pspice to show its feasibility.

In this paper, the equations of a chaotic system are presented which are megastable. In this system, oscillator terms are forced to blink during the time. The dynamical system is introduced in Section 2. This system, which is inspired by the simplest megastable equations, has uncountable attractors. Coexisting limit cycles, torus, and chaotic attractors are investigated in this model in Section 2. In Section 3, the complexity of the system is assessed. Finally, the results are concluded in Section 5.

\section{The Proposed System}

The proposed model is inspired by the simplest megastable model, which was introduced by Jafari et al. [55]:

$$
\dot{x}=-y, \dot{y}=0.1 x+\sin (y) \text {. }
$$

When $(-y)$ and $(0.1 x)$ on the right side of equations have the responsibility of making oscillations. Some examples of transient trajectories and attractors of equation (1) are plotted in Figure 1. The system's only equilibrium point is ( 0 , 0 ) in the center of the smallest limit cycle. Consequently, because other attractors have no equilibrium in their basin, they can be considered hidden attractors.

These equations are used as a platform to introduce the new method of forcing, which is referred to as forcing oscillation terms to blink. To make the oscillation terms blink, their coefficients should change during time. For this aim, time-varying functions are multiplied by the coefficient of the oscillatory terms. These time-variant functions should oscillate between zero (to turn off oscillatory terms for moments) and a positive threshold. In other words, these time-variant oscillatory functions should not have negative values. Note that if the coefficient of oscillation terms oscillates between a positive and a negative value, the trajectory's rotation direction changes repetitively. These repetitive changes in the direction of the oscillator can disturb its stability. Therefore, inspired from equation (1), the oscillator is designed based on the following equations:

$$
\dot{x}=-\cos ^{2}(w t) y, \dot{y}=0.1 \sin ^{2}(w t) x+A \sin (y) .
$$

When $\cos ^{2}(w t)$ and $\sin ^{2}(w t)$ are coefficients of the linear oscillatory terms, the power two for sin and cos functions cause the coefficients to not have negative values. Consequently, this coefficients' oscillation between 0 and 1 caused the blinking of the equations' oscillatory terms. This system's fixed point is only $(0,0)$. The system's Jacobean is 


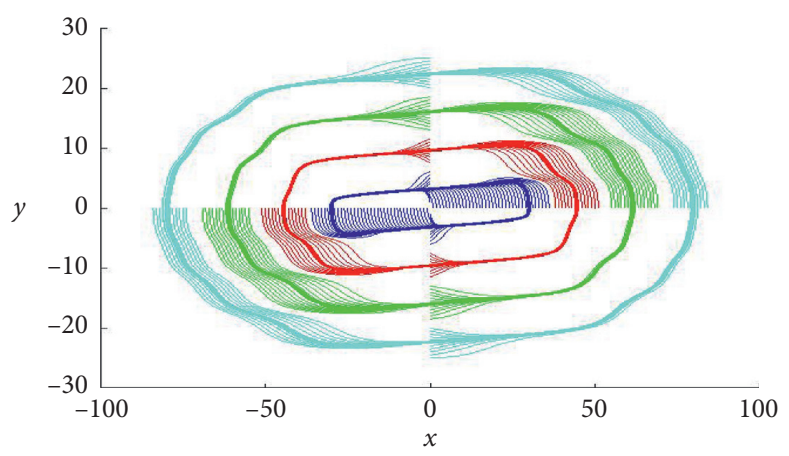

Figure 1: Attractor and some transient responses for equation (1). Attractors are plotted with thicker lines when transient responses are shown with thinner lines.

$$
J=\left[\begin{array}{cc}
0 & -\cos ^{2}(w t) \\
0.1 \sin ^{2}(w t) & A \cos (y)
\end{array}\right] \stackrel{(x, y)=(0,0)}{\longrightarrow}\left[\begin{array}{cc}
0 & -\cos ^{2}(w t) \\
0.1 \sin ^{2}(w t) & A
\end{array}\right]
$$

Therefore, the eigenvalues are

$$
\begin{aligned}
|\lambda I-J| & =0 \longrightarrow\left[\begin{array}{cc}
\lambda & +\cos ^{2}(w t) \\
-0.1 \sin ^{2}(w t) & \lambda-A
\end{array}\right] \stackrel{(x, y)=(0,0)}{\longrightarrow} \lambda^{2}-A \lambda+0.1 \cos ^{2}(w t) \sin ^{2}(w t)=0, \\
\lambda & =\frac{A \pm \sqrt{1-0.4 \cos ^{2}(w t) \sin ^{2}(w t)}}{2} .
\end{aligned}
$$

In this paper, always $A>1$ is considered. Paying attention that $0<\cos ^{2}(w t) \sin ^{2}(w t)<1$, the eigenvalues of the equilibrium are always positive. Therefore, the forced system always has an unstable equilibrium.

\section{Bifurcations and Lyapunov Exponents' Diagrams}

This work aims mainly to find possible chaotic behaviors in the proposed blinking system. Different compositions of $A$ and $w$ as the two bifurcation parameters may lead the system to chaotic behaviors. The ranges of these parameters are considered so that the system does not have unbounded solutions. Besides, ranges for the parameters have been presented in a way that system has different dynamical behaviors such as chaos, torus, and limit cycle. Therefore, firstly, $w=0.8$ is set, and the system's bifurcation is plotted for a range of $A$. Next, in the same way, $A=1$ is set, and a range for $w$ is investigated. Finally, by fixing $A=1$ and $w=$ 0.8 and using the initial value $\left(x_{0}, 0\right)$ as the bifurcation parameter, different coexisting attractors behaviors are investigated.

The Lyapunov exponents (LEs) and bifurcation diagrams are used as two powerful tools for investigating the system's behaviors for different parameters and initial conditions in this section. For all LEs diagrams, the smallest, which have the largest absolute value, is removed in the related pictures. It is done for better visualization of the other two.

Now, paying attention to Figure 2, the LE diagram shows chaotic behaviors for large ranges of $A$ values (one positive, one zero, and one negative (does not drown) [56]). Interestingly, by increasing the values of $A$, ranges that the system has a chaotic behavior become thinner; however, the values of the largest LEs increase.

Figure 2 shows LEs and bifurcation diagrams for a range of $w$. The LEs diagram in Figure 2 demonstrates chaotic solutions for different large ranges of $w$ values. Increasing the values of $w$, the length of ranges that the system has a chaotic behavior increases (Figure 3 ).

LEs and bifurcation diagrams (Figure 4) are plotted as a function of the initial condition $\left(x_{0}, 0\right)$. It can be seen that, for inner cycles, chaos can be detected. Besides, for the larger values of $x_{0}$, the system can present limit cycles (when one LE is zero and the two other is negative [56]) and torus (when the two largest LEs are zero and the other is negative [56]).

The system's detractors for different sets of parameters and different initial conditions are plotted in Figures 5 and 6. These attractors look like "picture frames." 


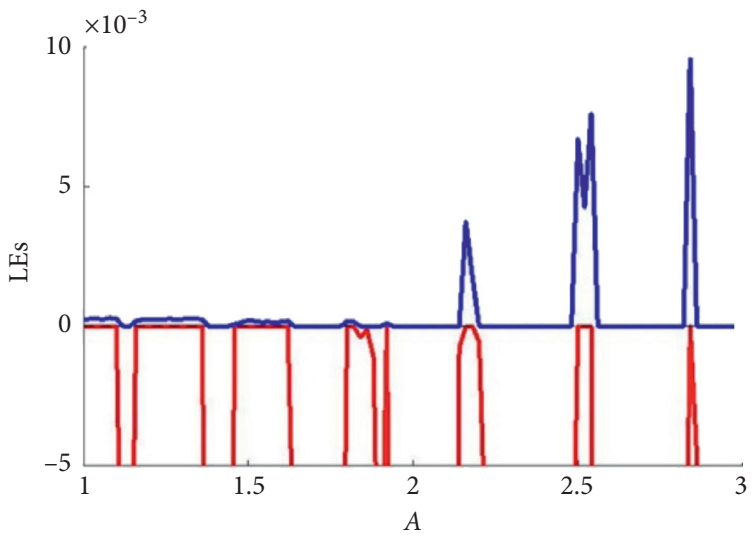

(a)

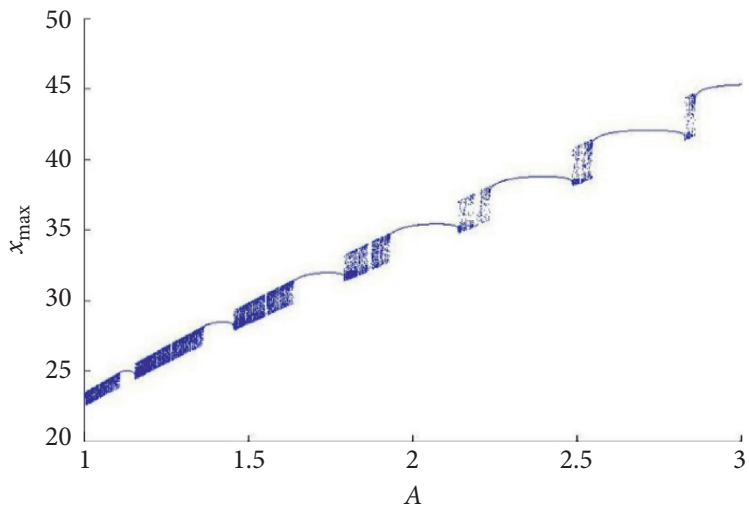

(b)

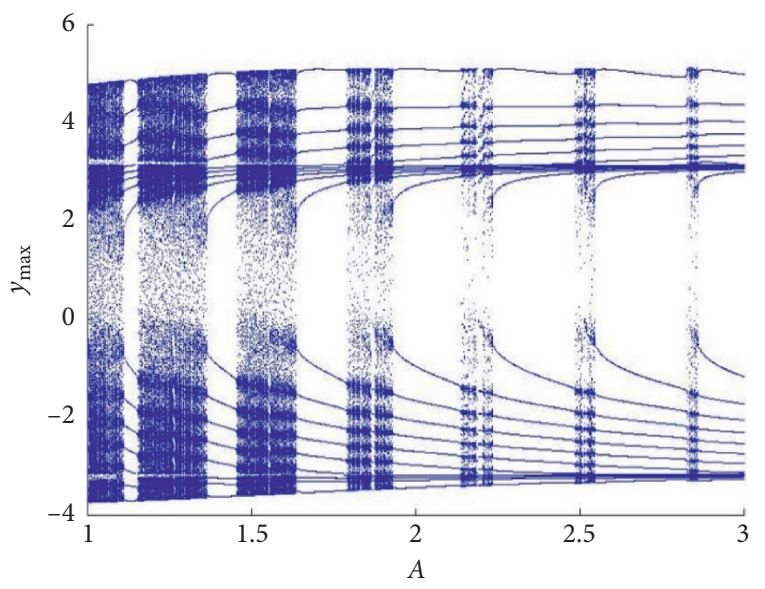

(c)

Figure 2: Lyapunov exponents and bifurcation diagrams are related to parameter $A$ for $w=0.8$ when the initial conditions are set $(0.01,0)$. (a) The two largest Lyapunov exponents are plotted and zoomed. Therefore, the third, which is negative (and has the largest absolute value), and also parts of the second cannot be seen for better visualization. (b) The local maximums of the $x$ variable time series. (c) The local maximums of the $y$ variable time series.

\section{Complexity-Based Sample Entropy Algorithm}

The sample entropy (SamEn) is a mathematical algorithm introduced to estimate the predictability of time series. It is usually used for evaluating how much information needs to predict the $(t+1)$ th output of a trajectory of systems using its previous $(t)$ outputs. Higher SamEn values indicate a dynamical system exhibits lower levels of regularity.

In this way, for a given time series $\{x(i)\}$, when $(i=0,1, \ldots, N-1)$, SamEn algorithm is given by the following [38].

(1) Reconstruct the time series to be as follows:

$$
X_{i}=\left\{x_{i}, x_{i+\tau}, \ldots, x_{i+(m-1) \tau}\right\}
$$

where $X_{i} \in R^{m}, m$ is the embedding dimension, and $\tau$ is the time delay.

(2) Compute the vector pairs for a given tolerance parameter $r$ by calculating the distance between $X_{i}$ and $X_{j}$ as follows:

$$
\begin{aligned}
d\left[X_{i}, X_{j}\right] & \leq r, d\left[X_{i}, X_{j}\right] \\
& =\max \{|x(i+k)-x(j+k)|: 0 \leq k \leq m-1\} .
\end{aligned}
$$

(3) Calculate $C_{i}^{m}(r)$, which represents the probability that any vector $X_{j}$ has a lower distance $(r)$ than $X_{i}$, as follows:

$$
C_{i}^{m}(r)=\frac{B_{i}}{N-(m-1) \tau},
$$

where $B_{i}$ is the number of vectors $X_{j}$ that have a lower distance $(r)$ than $X_{i}$.

(4) Obtain $\theta^{m}(r)$, which is the average of the natural logarithm of the function $C_{i}^{m}(r)$, as follows:

$$
\theta^{m}(r)=\frac{\sum_{i=1}^{N-(m-1) \tau} \ln C_{i}^{m}(r)}{[N-(m-1) \tau]}
$$

(5) Repeat the above steps to obtain $\theta^{m+1}(r)$, and then, calculate the SamEn as follows: 


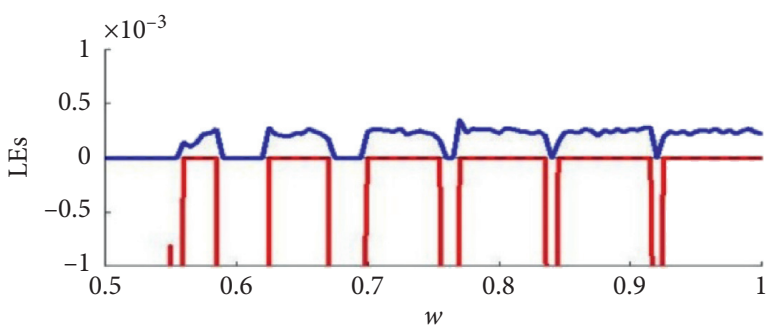

(a)

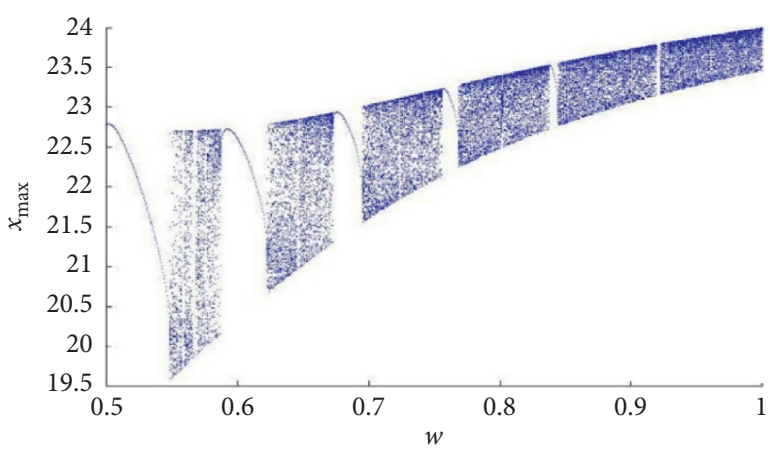

(b)

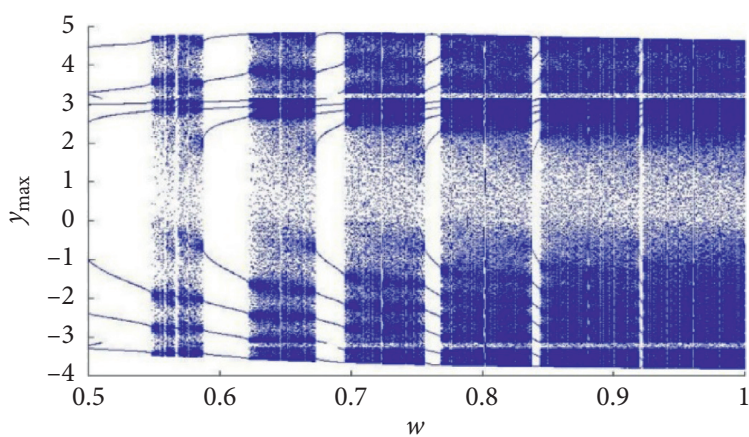

(c)

FigURE 3: Lyapunov exponents and bifurcation diagrams when $w$ changes as the bifurcation parameter. $A$ and initial conditions are set 1 and $(0.01,0)$, respectively. (a) The two largest Lyapunov exponents are shown and zoomed. Therefore, parts of the third, which is negative, and its absolute value is larger than the two others, are not plotted. Also, parts of the second Lyapunov exponent cannot be seen because of the zoom for better visualization. (b) Peaks of the $x$ variable time series. (c) Peaks of the $y$ variable time series.

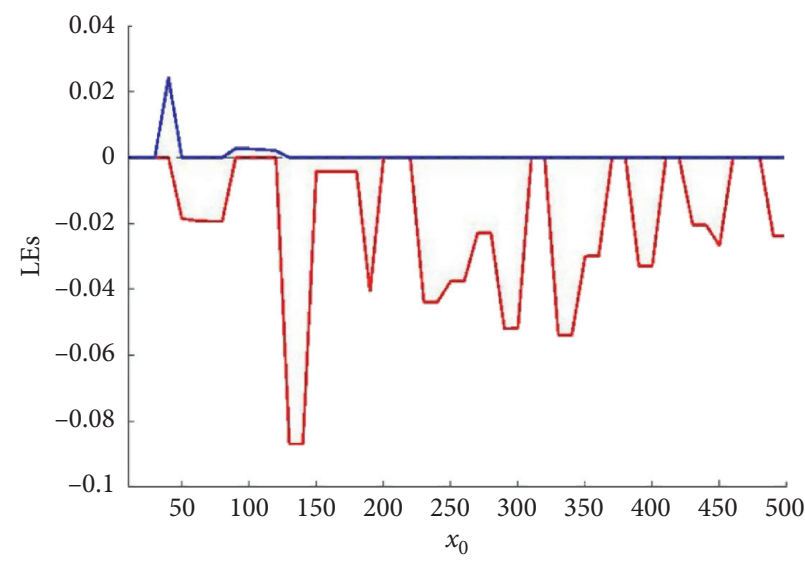

(a)

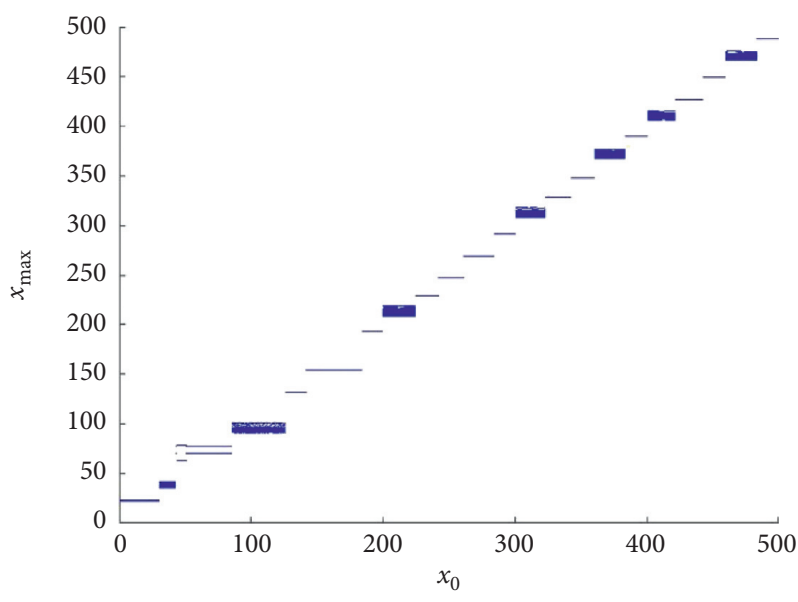

(b)

FIgURE 4: Continued. 


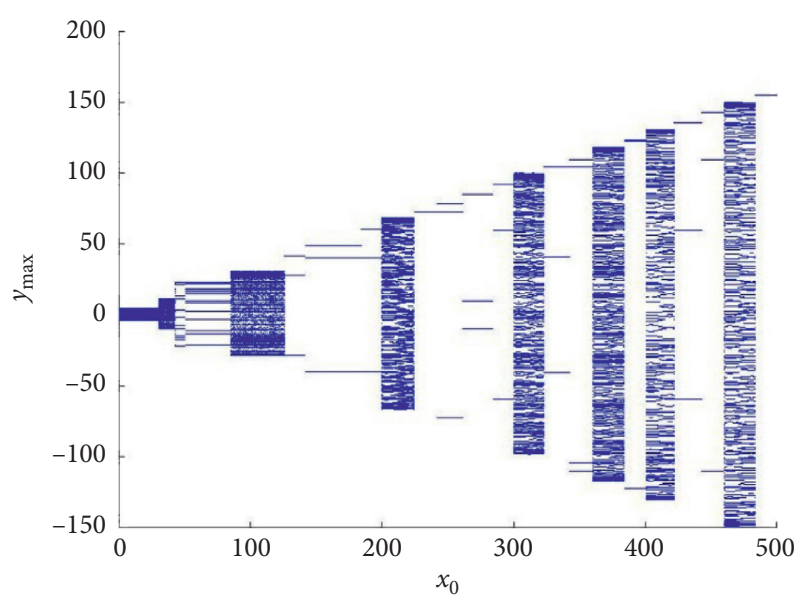

(c)

Figure 4: Lyapunov exponents and bifurcation diagrams for $A=1$ and $w=0.8$ when the initial conditions are set $\left(x_{0}, 0\right)$. (a) The two largest Lyapunov exponents are demonstrated. Therefore, the third, which is the most negative, is not shown. (b) $x$ time-series local maximums. (c) $y$ time-series local maximums.

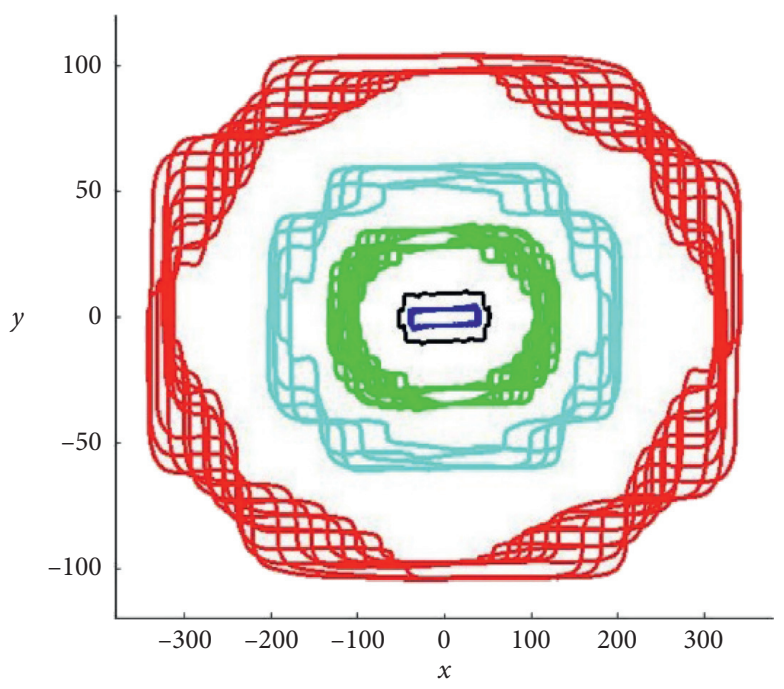

(a)

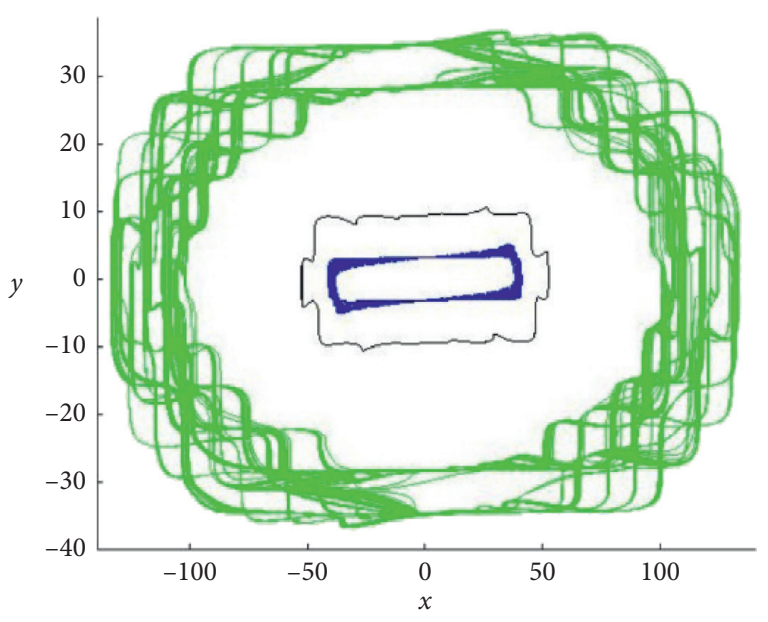

(b)

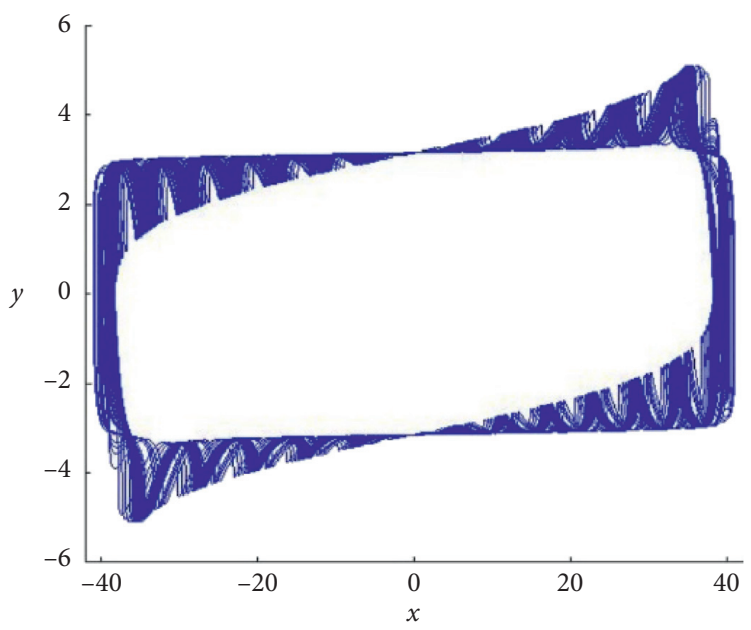

(c)

Figure 5: Trajectories when $A$ and $w$ are 2 and 0.5 , respectively. (a) The set initial values are $(0,2),(0,8),(0,30),(0,80)$, and $(0,100)$. (b and c) are zoomed versions of (a) for a better visualization. 


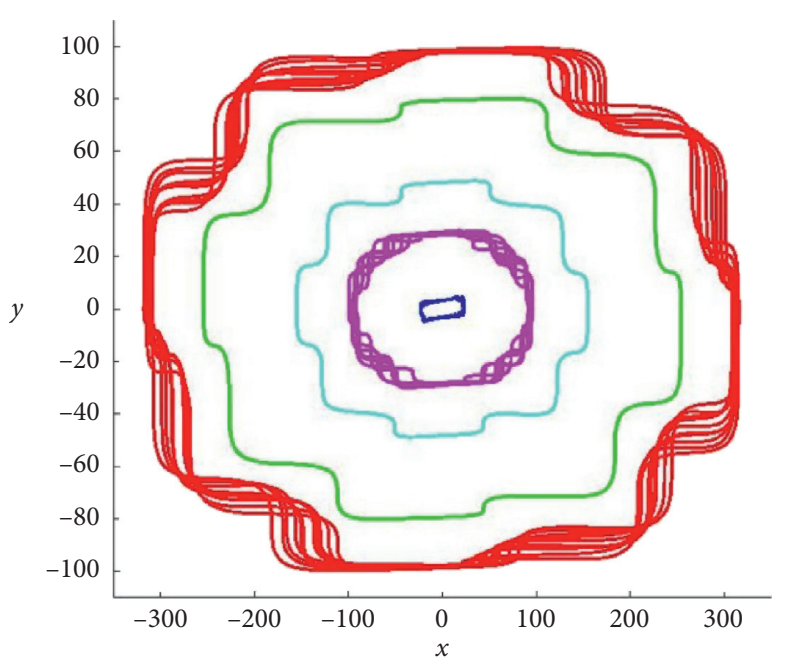

(a)

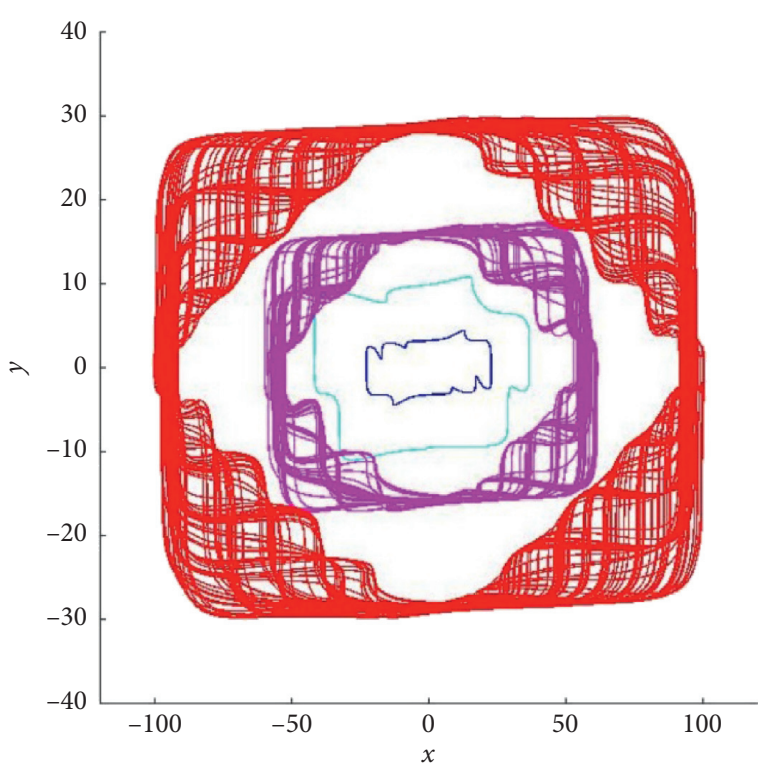

(b)

Figure 6: Trajectories for different sets of parameters and different initial values. (a) $A=2, w=0.5$, and the initial values are set $(0,2),(0,30),(0,45),(0,80)$, and $(0,150)$. (b) $A=1, w=0.5$, and the initial values are set $(0,2),(0,9),(0,15)$, and $(0,27)$.

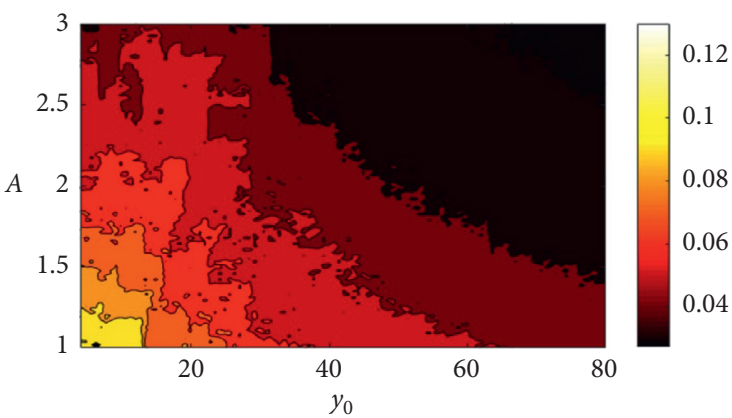

(a)

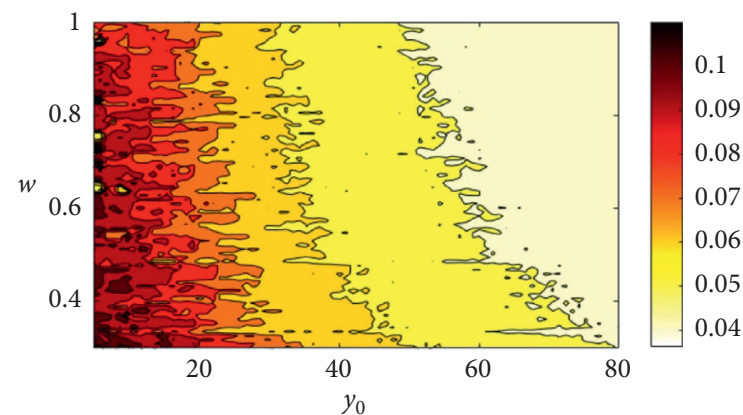

(b)

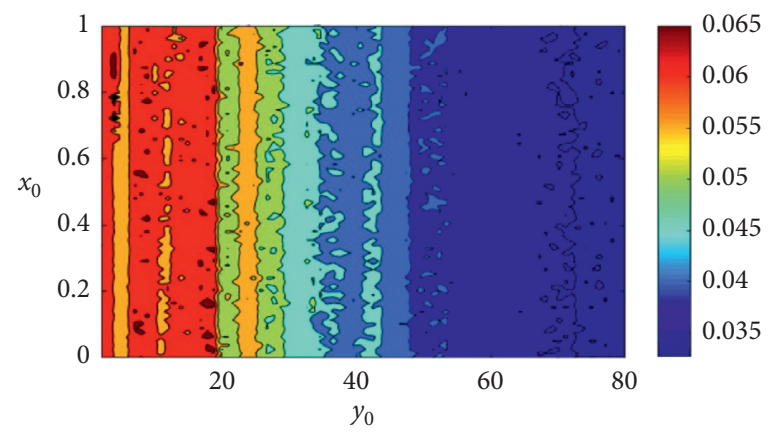

(c)

Figure 7: Complexity values of the system (equation (3)) calculated by SamEn. (a) $w=0.5$, and the initial values are set $\left(0, y_{0}\right)$. (b) $A=1$ and the initial values are set $\left(0.01, y_{0}\right)$. (c) $A=2, w=0.5$, and the initial values are set $\left(x_{0}, y_{0}\right)$.

$$
\operatorname{SamEn}(m, r, N)=\theta^{m}(r)-\theta^{m+1}(r) .
$$

Now, we employ the SamEn algorithm for $m=2$ and $r=0.2 \times$ (Standard Deviation) to evaluate the complexity of the multistability region of the megastable system (3). Figure 7(a) plots the SamEn values of the system when the parameter $A$ and the initial value $y_{0}$ change. As can be observed in this figure, when $A=1$, the system exhibits higher complexity values. The complexity values of the 

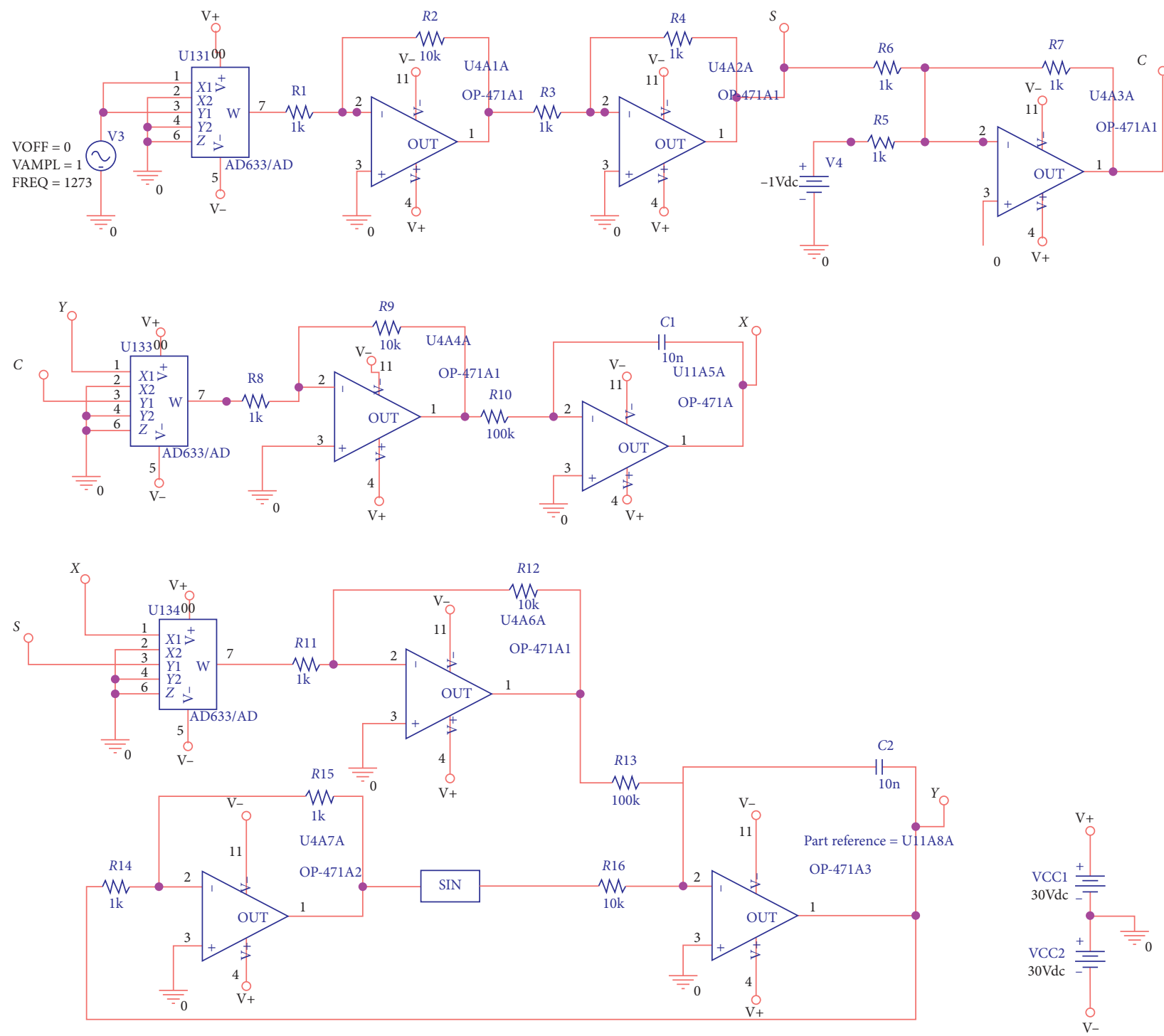

FIGURE 8: The circuit schematic of the introduced system (Pspice (version 9.1)). The elements values are chosen to represent the system when its parameters are considered $w=0.8$ and $A=1$. The system time is scaled $T=t / \alpha$ when $\alpha=(R=10 K \Omega) \times(C=10 \mathrm{nF})=10000$.

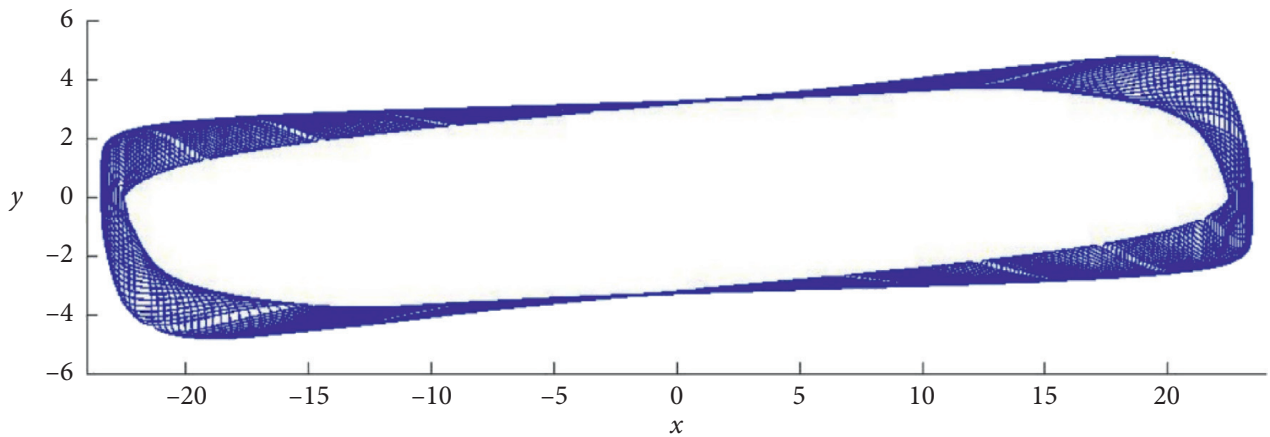

(a)

Figure 9: Continued. 


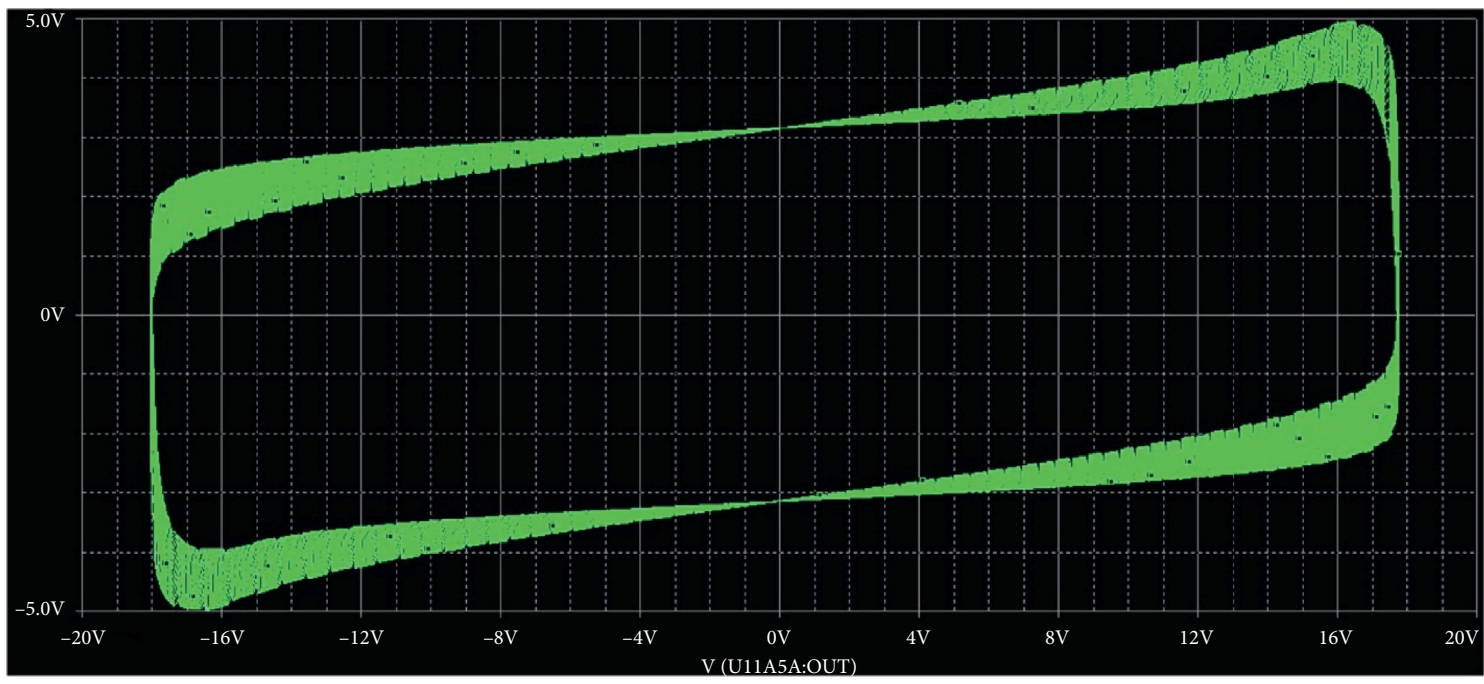

$\square \mathrm{V}(\mathrm{Y})$

(b)

FIgURE 9: The phase portrait of the simulated circuit. (a) The phase space of the system when $w=0.8$ and $A=1$ (simulated with Matlab 2020). (b) The result of the simulated circuit (Pspice, version 9.1).

system begin decreasing by increasing the value of $A$. Furthermore, Figure 7(b) shows the SamEn values for a range of $w$ and $y_{0}$. This figure demonstrates that the lower complexity values appear when the initial value $\left(y_{0}\right)$ increases. However, Figure $7(\mathrm{a})$ is also demonstrated this behavior.

To further visualize the system's complexity performance for a particular set of parameters, Figure $7(\mathrm{c})$ depicts its SamEn values when both initial values $\left(y_{0}\right.$ and $\left.x_{0}\right)$ change. This figure shows the complexity values of the system decrease when $\left(y_{0}\right)$ increases.

\section{Circuit Design}

The analog circuit of the introduced forced system is simulated in this part. The circuit is designed using simple elements such as resistors, capacitors, and Op-Amps (Figure 8$)$. To generate $\cos ^{2}(w t)$ and $\sin ^{2}(w t)$, an $\mathrm{AC}$ voltage source is used. AD633/AD is used to generate the second power of the AC voltage source. Considering AD633/AD multiply its outputs to 0.1 , the circuit of Op-Amp (U4A1A) (considering $R 1=1 \mathrm{k}$ and $R 2=10 \mathrm{k}$ ) is used for compensation. In the same way, Op_Amp (U4A4A) and Op-Amp (U4A6A) are used to compensate for the 0.1 coefficient of $\mathrm{AD} 633 / \mathrm{AD}$. Time is rescaled so that $T=t / \alpha$ when $\alpha=(R=$ $10 \mathrm{~K} \Omega) \times(C=10 \mathrm{nF})=10000$ is assumed. The equations of the implicated circuit are written as follows:

$$
\begin{aligned}
\cos ^{2}(w \alpha T) & =\left(\frac{R 7}{R 5}\right) 1-\frac{R 7}{R 6}\left(\frac{R 2 R 4}{R 1 R 3}\left(0.1 \sin ^{2}(w \alpha T) y\right)\right), \\
\frac{\mathrm{d} x}{\mathrm{~d}(\alpha T)} & =-\frac{1}{R 10 C 1}\left(\frac{R 9}{R 8}\right)\left(0.1 \cos ^{2}(w \alpha T) y\right), \\
\frac{\mathrm{d} y}{\mathrm{~d}(\alpha T)} & =\frac{1}{R 16 C 2}\left(\frac{R 12}{R 11} 0.1 \sin ^{2}(w \alpha T) x\right)+\frac{1}{R 16 C 2} \sin \left(\frac{R 15}{R 14} y\right) .
\end{aligned}
$$

When $x$ and $y$ represent the outputs of the Op-Amps U11A5A and U11A8A, respectively. The values of the elements are selected as follows: $R 1=1 K \Omega, R 2=1 K \Omega, R 3=$ $1 K \Omega, \quad R 4=1 K \Omega, \quad R 5=1 K \Omega, \quad R 6=1 K \Omega, \quad R 7=1 K \Omega$, $R 8=1 K \Omega, \quad R 9=1 K \Omega, \quad R 10=1 K \Omega, \quad R 11=1 K \Omega, \quad R 12=$ $1 K \Omega, R 13=1 K \Omega, R 14=1 K \Omega, R 15=1 K \Omega, R 16=1 K \Omega$,
$C 1=1 \mathrm{nF}$, and $C 2=1 \mathrm{KF}$. For $w=0.8$ the frequency of AC voltage is considered $(w=0.8) \times(\alpha=10000) / 2 \pi \approx 1273$. The system phase space simulated by Matlab for $w=0.8$ and $A=1$ is shown in Figure 9(a). Considering Figure 2, these parameters are chosen so that the system has a chaotic behavior. For the mentioned set of parameters, the circuit 
shown in Figure 8 is implicated in Pspice software (version 9.1). The result of the simulated circuit is demonstrated in Figure 9(b).

\section{Conclusion}

Considering recent interests in introducing new strange attractors, this paper introduced a new method to force oscillatory coupling terms of an oscillator. This method, which was named blinking forcing, considers oscillatory coefficients for oscillatory terms. The method was implicated on the simplest megastable system. The megastable forced dynamic could generate chaotic, torus, and limit cycle trajectories in its different attractors without changing its parameters. Complexity simulation analysis has demonstrated that these coexisting attractors exhibit different complexity values as the initial conditions vary. Besides, its "picture frame" like strange attractors can be considered a new topology that has not been proposed until yet, according to the authors' best knowledge. It is proposed that the introduced blinking method of forcing oscillatory terms is to be applied and studied on the other oscillators in the next research studies.

\section{Data Availability}

The data used to support the findings of the study are available within the article.

\section{Conflicts of Interest}

The authors declare that they have no conflicts of interest.

\section{Acknowledgments}

This work was partially funded by Center for Nonlinear Systems, Chennai Institute of Technology, India vide funding no. CIT/CNS/2021/RD/007.

\section{References}

[1] F. Nazarimehr and J. C. Sprott, "Investigating chaotic attractor of the simplest chaotic system with a line of equilibria," The European Physical Journal Special Topics, vol. 229, no. 6-7, pp. 1289-1297, 2020.

[2] Z. Faghani, F. Nazarimehr, S. Jafari, and J. C. Sprott, "Simple chaotic systems with specific analytical solutions," International Journal of Bifurcation and Chaos, vol. 29, no. 9, Article ID 1950116, 2019.

[3] G. A. Leonov and N. V. Kuznetsov, "Hidden attractors in dynamical systems. from hidden oscillations in hilbert-Kolmogorov, aizerman, and kalman problems to hidden chaotic attractor in chua circuits," International Journal of Bifurcation and Chaos, vol. 23, no. 1, Article ID 1330002, 2013.

[4] V.-T. Pham, S. Jafari, C. Volos, X. Wang, and S. M. R. H. Golpayegani, "Is that really hidden? The presence of complex fixed-points in chaotic flows with no equilibria," International Journal of Bifurcation and Chaos, vol. 24, no. 11, Article ID 1450146, 2014.

[5] Z. Wei, I. Moroz, J. Sprott, A. Akgul, and W. Zhang, "Hidden hyperchaos and electronic circuit application in a $5 \mathrm{D}$ self- exciting homopolar disc dynamo," Chaos: An Interdisciplinary Journal of Nonlinear Science, vol. 27, Article ID 033101, 2017.

[6] J. C. Sprott and B. Munmuangsaen, "Comment on "A hidden chaotic attractor in the classical Lorenz system"," Chaos, Solitons \& Fractals, vol. 113, pp. 261-262, 2018.

[7] M. Molaie, S. Jafari, J. C. Sprott, and S. M. R. H. Golpayegani, "Simple chaotic flows with one stable equilibrium," International Journal of Bifurcation and Chaos, vol. 23, no. 11, Article ID 1350188, 2013.

[8] S. Jafari and J. C. Sprott, "Simple chaotic flows with a line equilibrium," Chaos, Solitons \& Fractals, vol. 57, pp. 79-84, 2013.

[9] S. Jafari, J. C. Sprott, V.-T. Pham, C. Volos, and C. Li, "Simple chaotic 3D flows with surfaces of equilibria," Nonlinear Dynamics, vol. 86, no. 2, pp. 1349-1358, 2016.

[10] G. A. Leonov, N. V. Kuznetsov, M. A. Kiseleva, E. P. Solovyeva, and A. M. Zaretskiy, "Hidden oscillations in mathematical model of drilling system actuated by induction motor with a wound rotor," Nonlinear Dynamics, vol. 77, no. $1-2$, pp. 277-288, 2014.

[11] K. Sun and J. C. Sprott, "Periodically forced chaotic system with signum nonlinearity," International Journal of Bifurcation and Chaos, vol. 20, no. 5, pp. 1499-1507, 2010.

[12] B. Van der Pol, "On "relaxation-oscillations"," The London, Edinburgh, and Dublin Philosophical Magazine and Journal of Science, vol. 2, no. 11, pp. 978-992, 1926.

[13] B. Hernández-Galván, J. López-Rentería, B. AguirreHernández, and G. Fernández-Anaya, "Robust stability in discrete control systems via linear controllers with single and delayed time," Mathematical Problems in Engineering, vol. 2018, Article ID 3674628, 15 pages, 2018.

[14] E. Zambrano-Serrano, J. M. Muñoz-Pacheco, and E. CamposCantón, "Chaos generation in fractional-order switched systems and its digital implementation," AEU - International Journal of Electronics and Communications, vol. 79, pp. 43-52, 2017.

[15] N. R. Cazarez-Castro, M. Odreman-Vera, S. L. CardenasMaciel, H. Echavarria-Heras, and C. Leal-Ramírez, "Fuzzy differential equations as a tool for teaching uncertainty in engineering and science," Computación Y Sistemas, vol. 22, 2018.

[16] C. Li, J. C. Sprott, T. Kapitaniak, and T. Lu, "Infinite lattice of hyperchaotic strange attractors," Chaos, Solitons \& Fractals, vol. 109, pp. 76-82, 2018.

[17] J. R. Pulido-Luna, J. A. López-Rentería, and N. R. CazarezCastro, "Design of a nonhomogeneous nonlinear synchronizer and its implementation in reconfigurable hardware," Mathematical and Computational Applications, vol. 25, no. 3, p. 51, 2020.

[18] D. A. Prousalis, C. K. Volos, B. Bao, E. Meletlidou, I. N. Stouboulos, and I. M. Kyprianidis, "Extreme multistability in a hyperjerk memristive system with hidden attractors," in Recent Advances in Chaotic Systems and SynchronizationElsevier, Amsterdam, Netherlands, 2019.

[19] D. Prousalis, C. Volos, I. Stouboulos, I. Kyprianidis, and D. Frantzeskakis, "An extended study of extreme multistability in a memristive circuit," in Proceedings of the 2017 Panhellenic Conference on Electronics and Telecommunications (PACET). IEEE, 2017, pp. 1-4, Volos, Greece, May 2017.

[20] B. Pradhan, S. Mukherjee, A. Saha, H. Natiq, and S. Banerjee, "Multistability and chaotic scenario in a quantum pair-ion plasma," Zeitschrift für Naturforschung A, vol. 76, no. 2, pp. 109-119, 2021. 
[21] Y. Zhang and G. Luo, "Multistability of a three-degree-offreedom vibro-impact system," Communications in Nonlinear Science and Numerical Simulation, vol. 57, pp. 331-341, 2018.

[22] M. Chen, M. Sun, B. Bao, H. Wu, Q. Xu, and J. Wang, "Controlling extreme multistability of memristor emulatorbased dynamical circuit in flux-charge domain," Nonlinear Dynamics, vol. 91, no. 2, pp. 1395-1412, 2018.

[23] V. T. Pham, D. S. Ali, N. M. G. Al-Saidi, K. Rajagopal, F. E. Alsaadi, and S. Jafari, "A novel mega-stable chaotic circuit,” Radioengineering, vol. 29, no. 1, pp. 140-146, 2020.

[24] M. A. Rahim, H. Natiq, N. Fataf, and S. Banerjee, "Dynamics of a new hyperchaotic system and multistability," The European Physical Journal Plus, vol. 134, pp. 1-9, 2019.

[25] E. Jiménez-López, J. S. González Salas, L. J. Ontañón-García, E. Campos-Cantón, and A. N. Pisarchik, "Generalized multistable structure via chaotic synchronization and preservation of scrolls," Journal of the Franklin Institute, vol. 350, no. 10, pp. 2853-2866, 2013.

[26] A. Pisarchik, R. Jaimes-Reátegui, C. Rodríguez-Flores, J. García-López, G. Huerta-Cuellar, and F. Martín-Pasquín, "Secure chaotic communication based on extreme multistability," Journal of the Franklin Institute, vol. 358, no. 4, pp. 2561-2575, 2021.

[27] U. Feudel, A. N. Pisarchik, and K. Showalter, "Multistability and tipping: from mathematics and physics to climate and brain-minireview and preface to the focus issue," Chaos: An Interdisciplinary Journal of Nonlinear Science, vol. 28, Article ID 033501, 2018.

[28] W. J. Sanders, "The topology of chaos: alice in stretch and squeezeland," The Mathematics Teacher, vol. 96, p. 218, 2003.

[29] C. Li, W. Hu, J. C. Sprott, and X. Wang, "Multistability in symmetric chaotic systems," The European Physical Journal Special Topics, vol. 224, no. 8, pp. 1493-1506, 2015.

[30] H. Natiq, M. R. Kamel Ariffin, M. A. Asbullah, Z. Mahad, and M. Najah, "Enhancing chaos complexity of a plasma model through power input with desirable random features," Entropy, vol. 23, p. 48, 2021.

[31] E.-C. Díaz-González, B. Aguirre-Hernández, J. A. LópezRentería, E. Campos-Cantón, and C. A. Loredo-Villalobos, "Stability and multiscroll attractors of control systems via the abscissa," Mathematical Problems in Engineering, vol. 2017, Article ID 6743734, 19 pages, 2017.

[32] E. C. Díaz-González, J.-A. López-Rentería, E. Campos-Cantón, and B. Aguirre-Hernández, "Maximal unstable dissipative interval to preserve multi-scroll attractors via multisaturated functions," Journal of Nonlinear Science, vol. 26, no. 6, pp. 1833-1850, 2016.

[33] B. Aguirre-Hernández, E. Campos-Cantón, J. A. LópezRenteria, and E. C. Díaz González, "A polynomial approach for generating a monoparametric family of chaotic attractors via switched linear systems," Chaos, Solitons \& Fractals, vol. 71, pp. 100-106, 2015.

[34] G. Huerta-Cuellar, E. Jiménez-López, E. Campos-Cantón, and A. N. Pisarchik, "An approach to generate deterministic Brownian motion," Communications in Nonlinear Science and Numerical Simulation, vol. 19, no. 8, pp. 2740-2746, 2014.

[35] H. E. Gilardi-Velázquez and E. Campos-Cantón, "Nonclassical point of view of the brownian motion generation via fractional deterministic model," International Journal of Modern Physics C, vol. 29, no. 3, Article ID 1850020, 2018.

[36] J. C. Sprott, "Simplest dissipative chaotic flow," Physics Letters A, vol. 228, no. 4-5, pp. 271-274, 1997.

[37] H. Natiq, S. Banerjee, and M. R. M. Said, "Cosine chaotification technique to enhance chaos and complexity of discrete systems," The European Physical Journal Special Topics, vol. 228, no. 1, pp. 185-194, 2019.

[38] J. S. Richman and J. R. Moorman, "Physiological time-series analysis using approximate entropy and sample entropy," American Journal of Physiology-Heart Circulatory Physiology, vol. 278, pp. 2039-2049, 2000.

[39] H. Natiq, M. Said, M. Ariffin, S. He, L. Rondoni, and S. Banerjee, "Self-excited and hidden attractors in a novel chaotic system with complicated multistability," The European Physical Journal Plus, vol. 133, pp. 1-12, 2018.

[40] J. C. Sprott, "A new class of chaotic circuit," Physics Letters A, vol. 266, pp. 19-23, 2000.

[41] G. D. Leutcho, S. Jafari, I. I. Hamarash, J. Kengne, Z. Tabekoueng Njitacke, and I. Hussain, "A new megastable nonlinear oscillator with infinite attractors," Chaos, Solitons \& Fractals, vol. 134, Article ID 109703, 2020.

[42] E. Tlelo-Cuautle, A. Dalia Pano-Azucena, O. GuillénFernández, and A. Silva-Juárez, "Analog implementations of fractional-order chaotic systems," in Analog/Digital Implementation of Fractional Order Chaotic Circuits and ApplicationsSpringer, Berlin, Germany, 2020.

[43] E. Tlelo-Cuautle, A. Dalia Pano-Azucena, O. GuillénFernández, and A. Silva-Juárez, "Integer and fractional-order chaotic circuits and systems," in Analog/Digital Implementation of Fractional Order Chaotic Circuits and ApplicationsSpringer, Berlin, Germany, 2020.

[44] A. Silva-Juárez, E. Tlelo-Cuautle, L. G. De la Fraga, and R. Li, "FPAA-based implementation of fractional-order chaotic oscillators using first-order active filter blocks," Journal of Advanced Research, vol. 25, pp. 77-85, 2020.

[45] E. Tlelo-Cuautle, A. D. Pano-Azucena, O. Guillén-Fernández, and A. Silva-Juárez, Analog/digital Implementation of Fractional Order Chaotic Circuits and Applications, Springer, Berlin, Germany, 2020.

[46] V. Carbajal-Gómez, E. Tlelo-Cuautle, R. Trejo-Guerra, C. Sánchez-López, and J. Munoz-Pacheco, "Experimental synchronization of multiscroll chaotic attractors using current-feedback operational amplifiers," Nonlinear Science Letters B: Chaos, Fractal Synchronization, vol. 1, pp. 37-42, 2011.

[47] H. Chen, S. He, A. D. Pano Azucena et al., "A multistable chaotic jerk system with coexisting and hidden attractors: dynamical and complexity analysis, FPGA-based realization, and chaos stabilization using a robust controller," Symmetry, vol. 12, no. 4, p. 569, 2020.

[48] A. Sambas, S. Vaidyanathan, E. Tlelo-Cuautle et al., "A novel chaotic system with two circles of equilibrium points: multistability, electronic circuit and FPGA realization," Electronics, vol. 8, no. 11, p. 1211, 2019.

[49] A. Sambas, S. Vaidyanathan, E. Tlelo-Cuautle et al., "A 3-D multi-stable system with a peanut-shaped equilibrium curve: circuit design, FPGA realization, and an application to image encryption," IEEE Access, vol. 8, pp. 137116-137132, 2020.

[50] O. Guillen-Fernandez, A. Silva-Juarez, A. Melendez-Cano, J. C. Nunez-Perez, and E. Tlelo-Cuautle, "On the FPGA-based implementation of the synchronization of chaotic oscillators in master-slave topology*," 2019.

[51] A. Pano-Azucena, E. Tlelo-Cuautle, S. Tan, B. Ovilla-Martinez, and L. De la Fraga, "FPGA-based implementation of a multilayer perceptron suitable for chaotic time series prediction," Technologies, vol. 6, no. 4, p. 90, 2018.

[52] E. Tlelo-Cuautle, J. D. Díaz-Muñoz, A. M. González-Zapata et al., "Chaotic image encryption using hopfield and 
hindmarsh-rose neurons implemented on FPGA," Sensors, vol. 20, no. 5, p. 1326, 2020.

[53] E. Tlelo-Cuautle, O. Guillén-Fernández, J. De Jesus RangelMagdaleno, A. Melendez-Cano, J. C. Nuñez-Perez, and L. G. De la Fraga, "FPGA implementation of chaotic oscillators, their synchronization, and application to secure communications," in Recent Advances in Chaotic Systems and Synchronization, pp. 301-328, Elsevier, Amsterdam, Germany, 2019.

[54] E. Tlelo-Cuautle, A. Dalia Pano-Azucena, O. GuillénFernández, and A. Silva-Juárez, "Synchronization and applications of fractional-order chaotic systems," in Analog/ Digital Implementation of Fractional Order Chaotic Circuits and ApplicationsSpringer, Berlin, Germany, 2020.

[55] S. Jafari, K. Rajagopal, T. Hayat, A. Alsaedi, and V.-T. Pham, "Simplest megastable chaotic oscillator," International Journal of Bifurcation and Chaos, vol. 29, no. 13, Article ID 1950187, 2019.

[56] J. C. Sprott, Elegant Chaos: Algebraically Simple Chaotic Flows, World Scientific, Singapore, 2010. 